\title{
Efficacious pleurodesis with OK-432 and doxorubicin against malignant pleural effusions
}

\author{
K. Kishi*, S. Homma*, S. Sakamoto*, M. Kawabata*, E. Tsuboi*, K. Nakata\#, K. Yoshimura*
}

Efficacious pleurodesis with $O K-432$ and doxorubicin against malignant pleural effusions. K. Kishi, S. Homma, S. Sakamoto, M. Kawabata, E. Tsuboi, K. Nakata, K. Yoshimura. (C) ERS Journals Ltd 2004.

ABSTRACT: Malignant pleural effusion develops frequently in patients with advanced lung cancer. Chemical pleurodesis is the most effective palliative treatment for these patients.

The efficacy of pleurodesis using both OK-432, a preparation of Streptococcus pyogenes, and doxorubicin for 20 patients with cytology-proven malignant pleural effusion associated with lung cancer was evaluated. After complete removal of pleural effusion, OK-432 and $30 \mathrm{mg}$ of doxorubicin were injected via an inserted chest tube. Treatment was terminated when the volume of daily drainage reached $<\mathbf{2 0 0} \mathbf{~ m L}$. If the daily volume remained $>200 \mathrm{~mL}$, an additional OK-432 was administered every 3 days.

In total, 16 patients $(80 \%)$ revealed a complete response, two patients $(10 \%)$ revealed a partial response, and no response was seen in two patients. Eighteen patients with complete or partial responses did not show subsequent reaccumulation of pleural effusion after pleurodesis. The chest tube remained in place for an average of 6.4 days, draining a mean of $2,854 \mathrm{~mL}$. The main side-effects were fever and pain that were easily treated with nonsteroidal anti-inflammatory drugs.

Pleurodesis using both OK-432 and doxorubicin showed high efficacy for controlling malignant pleural effusions caused by lung cancer.

Eur Respir J 2004; 24: 263-266.
*Dept of Respiratory Medicine, Respiratory Center, Toranomon Hospital, and ${ }^{\#}$ Dept of Respiratory Medicine, Toho University School of Medicine, Tokyo, Japan.

Correspondence: K. Kishi

Dept of Respiratory Medicine

Respiratory Center

Toranomon Hospital

2-2-2 Toranomon, Minato-ku

Tokyo 105-8470

Japan

Fax: 81335827068

E-mail:kazumak@toranomon.gr.jp

Keywords: Doxorubicin

lung cancer

malignant pleural effusion

OK-432

pleurodesis

Received: December 122003

Accepted after revision: February 292004
Malignant pleural effusion develops frequently in patients with lung cancer. The presence of pleural effusion typically signals advanced lung cancer and is associated with poor prognosis. Progressive pleural effusion causes respiratory insufficiency and demands palliative management of the effusion. Chemical pleurodesis via chest tube is the most common and effective approach for these terminally ill patients [1]. For small cell lung cancer, pleurodesis is indicated only when chemotherapy is contraindicated or ineffective [1, 2].

Various chemical agents have been used in an attempt to produce pleurodesis. WALKER-RENARD et al. [3] reviewed articles in the English language from 1966 to 1992 describing patients with recurrent, symptomatic pleural effusions who were treated with chemical pleurodesis. They defined complete response as the absence of reaccumulation of the effusion determined by clinical examination or chest radiograph. The overall complete response rate to chemical pleurodesis was $64 \%$. The complete success rate with fibrosing agents (nonanticancer agents) was $75 \%$, compared with a complete success rate of only $44 \%$ for anticancer agents. Talc was the most effective agent, with a complete success rate of $93 \%$ [1-3].

In Japan, talc is not commercially available and OK-432 is widely used for chemical pleurodesis in clinical practice. OK432 is a preparation of Streptococcus pyogenes, type A3, which was developed in Japan as an immunotherapeutic agent for cancer [4]. Pleurodesis with OK-432 has been reported to be efficacious for controlling malignant pleural effusions [5, 6]. Moreover, a recent study suggests intrapleural injection of both OK-432 and an anticancer agent may be more beneficial than that of an anticancer agent or OK-432 alone [7]. There have been several reports using a combination of OK-432 and doxorubicin for controlling malignant pleural effusions in Japan including the authors' study [5, 7-9]. In the authors' previous study, which was conducted in an uncontrolled and retrospective design in 67 individuals with pleuritis carcinomatosa, the combination of OK-432 and doxorubicin had achieved a high response rate of $85 \%$ [9].

The present study was conducted to evaluate prospectively the efficacy of pleurodesis using a combination of OK-432 and doxorubicin for controlling malignant pleural effusions caused by lung cancer.

\section{Materials and methods}

A total of 20 patients with cytologically proven malignant pleural effusions associated with lung cancer were studied prospectively between June 2001 and June 2003. The criteria for patient enrollment into the study included: 1) malignant pleural effusion proven by cytological examination; 2) Eastern Cooperative Oncology Group performance status of $0-3$; 3) no previous chemotherapy, thoracic radiation therapy and/or surgery received within 4 weeks; 4) no concomitant systemic chemotherapy or radiation therapy; 5) progressive pleural effusion after platinum-containing chemotherapy in patients with small cell lung cancer; and 6) no history or evidence of penicillin allergy. Informed consent was obtained from all the patients enrolled on the study.

OK-432 is a penicillin-treated, lyophilised preparation of S. pyogenes, group A3 (Picibanil, Chugai Pharmaceutical 
Co. Ltd, Tokyo, Japan). The Klinische Einheit (KE) is used to express the dosage of preparation; $1 \mathrm{KE}$ of $\mathrm{OK}-432$ contains $0.1 \mathrm{mg}$ of dried cocci [10].

All patients were hospitalised and a 20 -French chest tube was inserted into the pleural space under local anesthesia. The tube was connected to a water-sealed drainage system. After complete removal of pleural effusion, $10 \mathrm{KE}$ of OK-432 and $30 \mathrm{mg}$ of doxorubicin (Adriacin; Kyowa-Hakko Co. Ltd, Tokyo, Japan) in $200 \mathrm{~mL}$ of physiological saline were injected via the tube. The chest tube was clamped and patients were repositioned every $30 \mathrm{~min}$ for $2 \mathrm{~h}$. Treatment was terminated when the volume of daily drainage reached $<200 \mathrm{~mL}$. If the daily volume remained $>200 \mathrm{~mL}$, an additional $5 \mathrm{KE}$ of OK432 was administered every 3 days.

Chest radiography was done weekly to assess the response of pleural effusion to treatment. Complete blood count, liver function test, and performance status were measured every week.

The treatment response was evaluated by chest radiograph or computed tomography (CT) with the following criteria: 1) complete response, no fluid accumulation for $>4$ weeks; 2) partial response, a marked decrease or no increase of pleural fluid for $>4$ weeks; 3 ) no response, reaccumulation of pleural fluid within 4 weeks. Toxicities were graded according to the National Cancer Institute common toxicity criteria [11].

After the patient was discharged, a follow-up check ensued at the outpatient clinic. Chest radiography was performed monthly to assess reaccumulation of the pleural effusion. The survival time from the date of pleurodesis was measured by the Kaplan-Meier method [12].

\section{Results}

Patients' characteristics are listed in table 1. There were 14 males and six females aged 30-84 yrs (mean 66.2 \pm 13.4 yrs). The performance status of $40 \%$ of patients was 2 or 3 . Seventeen patients had adenocarcinoma, two had small cell carcinoma and one had squamous cell carcinoma. The clinical

Table 1.-Patient characteristics

\begin{tabular}{lc}
\hline Characteristics & Patients \\
\hline N & 20 \\
Sex & \\
$\quad$ Male & 14 \\
$\quad$ Female & 6 \\
Age yrs & $66.2(30-84)$ \\
Performance status* & 12 \\
$\quad 1$ & 2 \\
2 & 6 \\
3 & 17 \\
Histological classification & 2 \\
$\quad$ Adenocarcinoma & 1 \\
Small cell carcinoma & \\
Squamous cell carcinoma & 13 \\
Clinical stage & 7 \\
IIIB & \\
IV & \\
Site of remote metastasis & 5 \\
$\quad$ Bone & 4 \\
Lung & \\
Prior treatment & \\
$\quad$ Chemotherapy & 7 \\
Surgical resection & 1 \\
Irradiation & 1 \\
\hline
\end{tabular}

Data are presented as $\mathrm{n}$ or mean (range); *: Eastern Cooperative Oncology Group.
Table 2. - Side-effects by pleurodesis using OK-432 and doxorubicin

\begin{tabular}{lccccc}
\hline & \multicolumn{3}{c}{ Grade } & Incidence \\
\cline { 2 - 4 } & 1 & 2 & 3 & 4 & $\%$ \\
\hline Fever & 9 & 5 & 0 & 0 & 70 \\
Chest pain & 3 & 4 & 0 & 0 & 35 \\
Appetite loss & 6 & 0 & 0 & 0 & 30 \\
sALT elevation & 1 & 3 & 0 & 0 & 20 \\
\hline
\end{tabular}

sALT: Serum alanine aminotransferase.

stage in 13 of 20 patients was IIIB, and seven of 20 were IV. The sites for remote metastasis were bone in five patients and lung in four. Prior treatment included chemotherapy in seven patients, surgery in one and irradiation in one, respectively.

The patients were followed up from 1 to 19.8 months with a mean period of 5.4 months. Nine patients died, four were still alive, and five were referred to another hospital during these follow-up periods.

Sixteen patients $(80 \%)$ revealed a complete response, two patients $(10 \%)$ revealed a partial response, and no response was observed in two patients. The rate of objective treatment response was $90 \%$. Among 18 patients with complete and partial responses, the time point of the last evaluation was at 1 month in four patients, 2 months in two, 3 months in two, 4 months in three, 5 months in three, 7 months in one and $>12$ months in three, respectively. No recurrence of pleural effusion was found in these patients who showed complete and partial responses during their whole follow-up periods. Eight patients received systemic chemotherapy after pleurodesis.

The number of intrapleural injections of OK-432 was one in 14 patients and two in six. The average number and dose of intrapleural injection of OK-432 was 1.3 times and $11.2 \mathrm{KE}$, respectively. The chest tube remained in place for an average of 6.4 days (range 3-12 days), and draining volume of $2,854 \mathrm{~mL}$ (range $830-5,814 \mathrm{~mL}$ ).

The side-effects by pleurodesis using OK-432 and doxorubicin are summarised in table 2. Grade 1 to 2 fever occurred in $70 \%$ of the patients. The mean duration of fever higher than $38.0^{\circ} \mathrm{C}$ was 2.2 days (range $0-7$ days). Grade 1 to 2 chest pain was documented in $35 \%$ of the patients. Both fever and chest pain were easily managed with nonsteroidal anti-inflammatory drugs. The level of serum alanine aminotransferase rose transiently in $20 \%$ of the patients. Grade 3 or 4 side-effects were not noted.

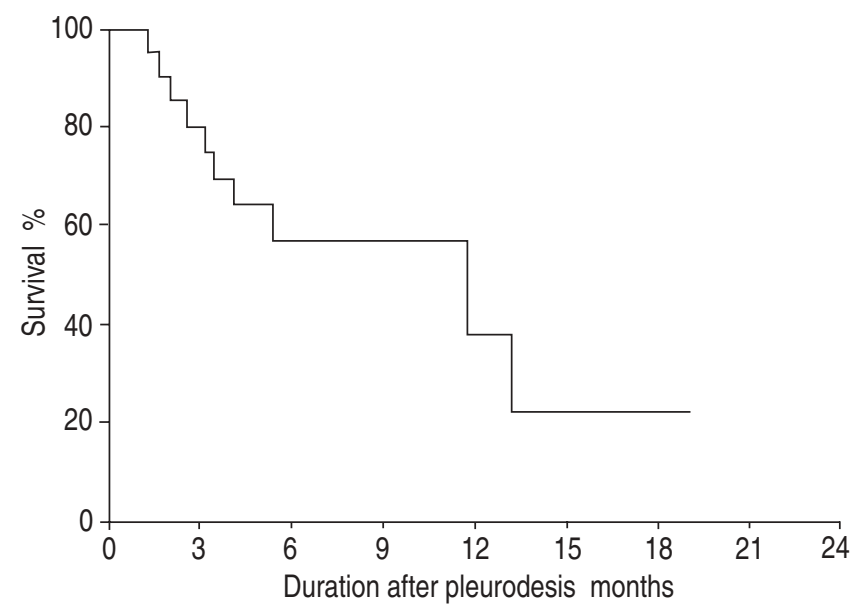

Fig. 1. - Survival curve of all patients. 
Table 3. - Previous studies on pleurodesis with combinations including OK-432 for malignant pleural effusions

\begin{tabular}{|c|c|c|c|c|c|}
\hline Study design & Sclerosing agents & Patients $\mathrm{n}$ & Observation period months & Success rate $\% \#$ & References \\
\hline Randomised, & OK-432+DXR & 29 & ND & 72 & [8] \\
\hline controlled study* & DXR & 24 & & 48 & \\
\hline $\begin{array}{l}\text { Retrospective, } \\
\text { uncontrolled study }\end{array}$ & OK-432+DXR & 67 & $6.0(1.1-26.5)$ & 85 & [9] \\
\hline $\begin{array}{l}\text { Retrospective, } \\
\text { uncontrolled study* }\end{array}$ & OK-432+MINO & 11 & $6.7(1.3-17.8)$ & 100 & [23] \\
\hline
\end{tabular}

Data are presented as n, mean (range) or per cent. DXR: doxorubicin; MINO: minocycline; ND: not described. *: patients other than lung cancer were included; \# : success rate included complete and partial responses.

The survival curve in all patients is shown in figure 1 . The median survival time was 4.3 months and 1-yr survival was $35.7 \%$.

\section{Discussion}

OK-432 is a preparation of $S$. pyogenes A3 and this agent has been used as an antitumour immunomodulator for lung, gastric and other cancers [13-16]. Further, a series of studies have demonstrated that $\mathrm{OK}-432$ is efficacious as a single agent in controlling malignant pleural effusion as well as malignant ascites $[5,6,17,18]$. Because of inaccessibility to talc in Japan, OK-432 has been widely used in pleurodesis for spontaneous pneumothorax or malignant pleural effusions [19]. This agent has a sclerosing effect that could induce pleural adhesion and obliterate the pleural cavity air space. The exact mechanisms of pleurodesis achieved by OK-432 are not clear. However, it is speculated that through its irritating effect, OK-432 would cause release of fibroblast stimulation factors to be released from activated neutrophils and lymphocytes [18]. In addition, direct cytotoxic and cytostatic effects of OK-432 on tumour cells have been reported [10, 20]. These antitumour effects may be due to an immunomodulatory property of OK-432, by activating neutrophils, macrophages, natural killer cells, or T-lymphocytes [21, 22]. Further, activated mononuclear cells are known to produce a variety of cytokines including interleukin-6, thus resulting in augmentation of antitumour effects [7].

In the present study, it has been demonstrated that pleurodesis with a combination of OK-432 and doxorubicin achieved a response rate of $90 \%$. Although the current study was an open and uncontrolled study and consisted of small group of patients, the obtained results of a high success rate for pleurodesis using OK-432 in combination with doxorubicin were very promising. In contrast, the use of intrapleural doxorubicin alone with the dosage of $10-40 \mathrm{mg}$ produced a complete response in $24 \%$ of the cases of malignant pleural effusion [3]. In addition, a randomised trial on the comparative effects of intrapleural OK-432 and mitomycin $\mathrm{C}$ administration for malignant pleural effusion caused by lung cancer revealed that pleurodesis with OK-432 achieved a higher complete response rate $(73 \%)$ than pleurodesis with mytomycin C (41\%) [6]. More importantly, OK-432 can be administered into the pleural or peritoneal cavity with anticancer agents. Simultaneous administration of OK-432 and one of the anticancer agents such as cisplatin, mitomycin $\mathrm{C}$ or doxorubicin achieved both a higher response rate and survival rate than that accomplished by any anticancer agent alone or OK-432 alone, according to the report of a randomised study for malignant effusions [7]. To date, there has been no prospective study conducted to evaluate the effect of pleurodesis with a combination of OK-432 and doxorubicin for malignant pleural effusions associated with lung cancer. The results of previous studies with a combination including OK-432 are shown in table 3 [8, 9, 23]. Although the therapeutic methods applied in each study were different, pleurodesis using OK-432 showed a high success rate in general, as was achieved in the present study.

Only two patients developed reaccumulation of pleural effusion within 4 weeks after pleurodesis in the present study. One patient had a moderate amount of pleural effusion for 7 months before being referred to Toranomon Hospital, Japan, and the other patient had a small amount of pleural effusion seen on CT over 2 yrs while receiving chemotherapy. In both patients, full lung expansion was not achieved after drainage. Therefore, early intervention is likely needed for successful pleurodesis for malignant pleural effusion.

The main side-effects in the present study were fever and chest pain of grade 1 or 2 . Fever was seen in $70 \%$ of the patients, but the mean duration of the fever was only 2 days. Both fever and chest pain were easily treated with nonsteroidal anti-inflammatory drugs. Although pleurodesis with OK-432 was previously reported to be associated with high complication rate $[1,6]$, the present study has demonstrated that this agent can be used safely without serious sideeffects even in patients who show a poor performance status of 2 or 3 .

The authors conclude that pleurodesis with a combination of OK-432 and doxorubicin is very efficacious for controlling malignant pleural effusions. Randomised studies are needed to find the optimal combination regimens.

\section{References}

1. Kvale PA, Simoff M, Prakash UBS. Palliative care. Chest 2003; 123: 284S-311S.

2. American Thoracic Society. Management of malignant pleural effusions. Am J Respir Crit Care Med 2000; 162: 1987-2001.

3. Walker-Renard PB, Vaughan LM, Sahn SA. Chemical pleurodesis for malignant pleural effusions. Ann Intern Med 1994; 120: 56-64.

4. Okamoto H, Shoin S, Koshimura S, Shimizu R. Studies on the anticancer and streptolysin S-forming abilities of hemolytic streptococci. Jpn J Microbiol 1967; 11: 323-336.

5. Reshad K, Inui K, Takeuchi Y, Takahashi Y, Hitomi S. Treatment of malignant pleural effusion. Chest 1985; 88: 393-397.

6. Luh K-T, Yang P-C, Kuo S-H, Chang D-B, Yu C-J, Lee L-N. Comparison of OK-432 and mitomycin C pleurodesis for malignant pleural effusion caused by lung cancer. A randomized trial. Cancer 1992; 69: 674-679.

7. Nio Y, Nagami H, Tamura K, et al. Multi-institutional randomized clinical study on the comparative effects of intracavital chemothrapy alone versus immunotherapy alone versus immunochemotherapy for malignant effusion. $\mathrm{Br}$ J Cancer 1999; 80: 775-785.

8. Urata A, Nishimura M, Ota K. Randomized controlled 
study of OK-432 in the treatment of cancerous pleurisy. Gan To Kagaku Ryoho 1983; 10: 1467-1503.

9. Kishi K, Kawabata M, Tsuboi E, et al. Pleurodesis with a combination of OK-432 and adriamycin for malignant pleural effusions caused by lung cancer. Haigan 1999; 39: 261-266.

10. Sakurai Y, Tsukagoshi S, Sato H, Akiba T, Suzuki S. Tumor-inhibitory effect of a streptococcal preparation (NSC-B116209). Cancer Chemother Rep 1972; 56: 9-17.

11. National Cancer institute. NCIC-CTC grading system. http:// ctep.cancer.gov/forms/CTCv20 4-30-992.pdf. Date updated: 30th April 1999; Date accessed: 1st April, 2000.

12. Kaplan EL, Meier P. Nonparametric estimation from incomplete observations. J Am Stat Assoc 1958; 53: 457-481.

13. Kimura I, Ohnishi T, Yasuhara S, Sugiyama M, Urabe Y. Immunochemotherapy in human lung cancer using the streptococcal agent OK-432. Cancer 1976; 37: 2201-2203.

14. Tokai Cooperative Study Group for Adjuvant ChemoImmunotherapy of Stomach Cancer. A controlled study of maintenance chemoimmunotherapy vs immunotherapy alone immediately following palliative gastrectomy and induction chemoimmunotherapy for advanced gastric cancer. Cancer Chemother Pharmacol 1981; 7: 5-10.

15. Watanabe Y, Iwa T. Clinical value of immunotherapy with the streptococcal preparation OK-432 in non-small cell lung cancer. J Bio Res Mod 1987; 6: 169-180.

16. Maehara Y, Sugimachi K, Akagi M, Kakegawa T, Shimazu H, Tomita M. Early postoperative chemotherapy following noncurative resection for patients with advanced gastric cancer. Br J Cancer 1992; 65: 413-416.

17. Torisu M, Katano M, Kimura Y, Itoh H, Takesue M. New approach to management of malignant ascites with a streptococcal preparation OK-432: I. Improvement of host immunity and prolongation of survival. Surgery 1983; 93: 357-364.

18. Katano M, Torisu M. New approach to management of malignant ascites with a streptococcal preparation OK-432: II. Intraperitoneal inflammatory cell-mediated tumor cell destruction. Surgery 1983; 93: 365-373.

19. Ishihara K, Hasegawa T, Okazaki M, et al. OK432 chemical pleurodesis as a standard therapy of spontaneous pneumothorax. Nihon Kyobu Shikkan Gakkai Zasshi 1988; 26: 10 15.

20. Nio Y, Zighelboim J, Berek JS, Bonavida B. Cytotoxic and cytostatic effects of the streptococcal preparation OK-432 and its subcellular fractions on human ovarian tumor cells. Cancer 1989; 64: 434 441.

21. Katano M, Torisu M. Neutrophil-mediated tumor cell destruction in cancer ascites. Cancer 1982; 50: 60-68.

22. Fujimura T, Torisu M. Neutrophil-mediated tumor cell destruction in cancer ascites: II. OK-432 attacks killer neutrophils through activation of component C5. Clin Immunopathol 1987; 43: 174-184.

23. Tanaka A, Sato T. Adhesion therapy for malignant pleural effusion (Intrapleural administration of OK-432 with minocycline). Nihon Kokyuki Gakkai Zasshi 1999; 37: 531-537. 